Revista Científica Signos Fónicos, 6(2): 1-23. Diciembre 2020, ISNN 2422-1716.

ARTÍCULO ORIGINAL

\title{
Percepción de los docentes sobre el diseño de estrategias de intervención fonoaudiológica indirecta para la lectoescritura
}

\section{Perception of the teachers on the design of strategies of indirect phono-idiological intervention for reading and writing}

Aguilar-Cañas, Sandra ${ }^{1}$; Ayala-Nieto, Patricia²; Bautista-Cárdenas, A3 Rojas-Lemus, Nahomy-Sirley ${ }^{3}$; Tamara-Moncada, Viviana-Andrea ${ }^{3}$.

Como citar este artículo: Aguilar-Cañas, Sandra; Ayala-Nieto, Patricia; Bautista-Cárdenas, Angelica; Rojas-Lemus, Nahomy-Sirley; Tamara-Moncada, Viviana-Andrea. Percepción de los docentes sobre el diseño de estrategias de intervención fonoaudiológica indirecta para la lectoescritura. Revistas Científica Signos Fónicos. 2020: 6 (2).1-23.

Recibido: mayo 20, 2020 Aprobado: agosto 23, 2020.

\section{RESUMEN}

INTRODUCCION: La presente investigación recopila la información obtenida sobre la percepción de los docentes de los grados primero y segundo sobre el diseño de estrategias de intervención fonoaudiológica indirecta para la lectoescritura de los escolares de una institución educativa pública. MÉTODOS: esta investigación es de tipo analítica con corte transversal y con metodología acción participación, la recolección de la información se realizó a través de la entrevista estructurada y un meta-plan, los resultados fueron analizados siguiendo la teoría fundamentada, a través del software Atlas Ti v7. RESULTADOS: De las encuestas aplicadas a los docentes, emergen 10 códigos In vivo y 30 de constructo social, de esta manera, se realiza el análisis agrupándolos en 2 categorías, primero y segundo de primaria. ANÁLISIS Y DISCUSIÓN: la percepción de los docentes indica un máximo nivel de satisfacción con respecto a las estrategias planteadas para la lectoescritura porque es un proceso para el aprendizaje y a su vez, un sistema de comunicación y meta cognición que se aplica a la escolaridad. El fonoaudiólogo como actor principal para el desarrollo de la lectura y la escritura, cumple el rol de colaborador con los docentes en el contexto escolar. CONCLUSIONES: La percepción de los docentes con respecto al diseño de estrategias de intervención fonoaudiológica indirecta tiene un nivel máximo de satisfacción ya que cumple con los criterios establecidos según los derechos básicos de aprendizaje y las distintas rutas con base teórica que favorecen la lectoescritura.

PALABRAS CLAVE: intervención indirecta, estrategias, lectura, escritura y docentes.

\footnotetext{
${ }^{1}$ Fonoaudiología, Maestría en Educación, Sandra_22@hotmail.com, Orcid: https://orcid.org/00000002-4248-3771, Fundación Virgilio Barco - Cúcuta, Colombia.

${ }^{2}$ Fonoaudiología, Especialista Práctica pedagógica, angelapatricia37@gmail.com, Orcid: orcid.org/0000-0002-0004-5191, Somefyr SAS- Cúcuta, Colombia.

${ }^{3}$ Practicantes.
} 


\begin{abstract}
INTRODUCTION: This research compiles the information about the perception of the teachers of the first and second grades about the design of indirect phono audiological intervention strategies for the reading of the students of a public educational institution. METHODS: this investigation is of analytical type with cross-section and with method of action, the collection of the information is done through the structured interview and a meta-plan, the results are analyzed following the fundamental theory, through the software Atlas. Ti v7. RESULTS: From the surveys applied to the documents, 10 In vivo codes and 30 social construction codes emerge, in this way, the analysis is grouped into 4 categories, reading and writing according to each grade. ANALYSIS AND DISCUSSION: the perception of teachers indicates a maximum level of satisfaction with respect to the strategies proposed for reading and learning, the communication system and the metacognition that is applied to schooling. The role of the main actor for the development of reading and writing fulfills the role of collaborator with teachers in the school context. CONCLUSIONS: The perception of the documents with respect to the design of speech therapy strategies has a maximum level of satisfaction that meets the learning principles and the different routes based on theory that favor reading and writing.
\end{abstract}

KEY WORDS: indirect intervention, strategies, learning, reading, writing and teacher.

\title{
INTRODUCCIÓN
}

Las funciones cognoscitivas juegan un papel fundamental dentro del desarrollo intelectual, emocional y estético del individuo, integrando de este modo una función y aspecto complejo del ser humano el cual es el lenguaje, siendo de gran importancia ya que contribuye en la preparación del mundo adulto permitiendo que las personas se comuniquen efectivamente (1).

En este panorama, se ha constituido una oportunidad para que los fonoaudiólogos expertos en competencia comunicativa reflejen su quehacer y lo operacionalicen en la vida de todos los integrantes de las comunidades educativas, estudiantes, padres, maestros y directivos y de esta forma usar asertivamente los instrumentos de significación como: hablar, escuchar, leer y escribir (1).

Lo anterior, unido a la consideración de la comunicación como un determinante de la calidad de vida de las personas, comprometió a la profesión de fonoaudiología con la promoción del bienestar comunicativo (2).

La meta cognición en el ámbito escolar, le permite al alumno seleccionar entre sus conocimientos, el más adecuado para utilizarlo posteriormente como estrategia de organización de un contenido y evaluar el resultado este mismo (3).

Entre dichas competencias comunicativas se incluyen la lectura y escritura como herramientas básicas de aprendizaje, estas permiten desarrollar las habilidades lingüísticas necesarias para aprender (4).

En el proceso de desarrollo de la lectoescritura, es importante establecer el nivel de dominio de la lengua escrita, procesamiento de la información a través de procesos perceptivos, léxicos, sintácticos, semánticos, etc. Además, la fase de automatización de la lectura, búsqueda del significado para comprender el texto, estrategias cognitivas y metacognitivas (conciencia de los propios procesos cognitivos y regulación de la cognición) (5), (6), (7).

El contexto escolar inclusivo sitúa a la Fonoaudiología ante el resto, como aquella área de la salud que se adapta a la realidad escolar para dar solución a las dificultades que presentan los estudiantes en el 
Aguilar, Ayala et al. Revista Científica Signos Fónicos, 2020, 6(2): 1-23

aprendizaje, procesos comunicativos del hombre y sus desordenes (8) mediante la elaboración de un modelo de intervención indirecta con una orientación coherente a los planteamientos propios de la escuela.

Este modelo de intervención y apoyo escolar requiere de una serie de cambios que representan un mayor acercamiento al aula, al currículum, a los docentes y, en definitiva, a las situaciones naturales en las que se manifiestan las dificultades en la Lecto-escritura basándose que en este ambiente el infante se enfrenta a diversas situaciones donde se ve implícita la comunicación y la lengua escrita (9), (10).

Es allí donde se plantea desde rol fonoaudiológico una propuesta de intervención indirecta basándose en que esta intervención involucra al cuerpo docente como autor principal desde un entorno natural, cabe resaltar, que el docente actúa como mediador para potenciar el rendimiento del estudiante, favorecer hábitos de estudio, fomentar la meta cognición para aprender a aprender y facilitar el aprendizaje significativo siguiendo los modelos y estrategias adecuados (11), (12).

Un modelo de apoyo curricular dirigido a la prevención de las dificultades en el aprendizaje de la lectura y la escritura en el aula, la cual debe considerar los siguientes aspectos:

En primer lugar, la intervención en el contexto escolar debe ser una labor compartida donde la cooperación y el trabajo en equipo entre el docente y el fonoaudiólogo son elementos esenciales, puesto que son profesionales en conexión que buscan un avance en cualquier proceso dentro de su vida escolar para cualquier estudiante, este compromiso compartido conlleva a la necesidad de desarrollar una acción educativa de aprendizaje exitoso (13).

Así mismo, la colaboración entre docentes representa una estrategia fundamental para garantizar el éxito en la implementación y desarrollo del diseño de las estrategias de intervención tomando principalmente el aula como contexto natural de aplicación de las mismas y en segundo lugar, debe estructurarse como una intervención preventiva, comunitaria e integral teniendo en cuenta los estándares básicos de competencias, que permita a la escuela ofrecer una respuesta exitosa de enseñanza y aprendizaje de las habilidades escolares (9),(14).

Ahora bien, para solucionar los paradigmas del aprendizaje de la lectoescritura en el contexto educativo, se utiliza la metódica representada por la investigación-acción participativa con el fin de guiar los procedimientos en este campo (15) y transformar la practica social y/o educativa para articular la investigación desde el ámbito fonoaudiológico con los docentes de primero y segundo de primaria.

La Investigación-Acción-Participación, se entiende como un enfoque investigativo de intervención social que busca recoger demandas de los actores implicados con el objetivo de transformar la realidad existente a través de procesos que faciliten la apropiación de recursos como redes, propuestas y acciones. Asimismo, busca apropiarse del conocimiento por parte de la comunidad a través de herramientas para su análisis y autodiagnóstico (16).

De acuerdo al tema de interés para la construcción de las estrategias más adecuadas para la población, en una primera instancia se realiza una revisión de artículos mediante la metodología PICO, la cual proporciono el sustento teórico para la elaboración y diseño de una cartilla con estrategias de intervención indirecta, la cual tuvo como fin ser una herramienta facilitadora de enseñanza de la lectura y escritura.

Esta primera parte de la investigación correspondió a un proyecto educativo creado por estudiantes en formación y docentes de prácticas formativas del programa de Fonoaudiología; el cual buscaba prevenir las dificultades en la adquisición de la lectoescritura desde el contexto natural sin ver afectado el Revista Científica Signos Fónicos, 2020, 6 (2): 1-23. ISNN 2422-1716. 
Aguilar, Ayala et al. Revista Científica Signos Fónicos, 2020, 6(2): 1-23

desarrollo continuo de una clase formal dentro de la institución solo apoyando de manera didáctica y completa el proceso dentro del aula de clase de la lectoescritura, fundamentado bajo un enfoque neuropsicológico (17), neuro lingüístico (3), Psico lingüístico (18) y meta cognitivo (19) expuesto por Fernando Cuetos, Alexander Luria, Kenneth Goodman e Isabel Solé, lo cual permitió la consolidación de la misma como propuesta didáctica y pedagógica.

De igual manera para la estructuración del diseño se tuvo en cuenta las disposiciones conceptuales y lineamientos establecidos por el Ministerio de Educación Nacional para cada una de las áreas y grados que se tienen en cuenta en este estudio (20), (21).

Teniendo en cuenta, esto y dando continuidad a un proceso de investigación es necesario continuar con una segunda fase del proyecto, la cual surgió de la necesidad de identificar y establecer las estrategias que más se adaptaran al entorno escolar teniendo en cuenta la perspectiva de los docentes de primero y segundo de primaria, debido a esto, se plantea el siguiente interrogante y pregunta de investigación:

¿Cuál es la percepción de los docentes acerca el diseño de estrategias de intervención indirecta para la lectura y la escritura?

\section{MÉTODOS}

Esta es una investigación de enfoque mixto (22), (23) de corte transversal (24), bajo una metodología de acción participación que supone la simultaneidad del proceso de conocer y de intervenir, e implica la participación de la misma gente involucrada en el programa de estudio y de acción (25), (26).

Este estudio, se llevó a cabo en una institución educativa del municipio de Villa del Rosario, Norte de Santander durante el periodo abril-julio de 2019. La población es de 12 docentes pertenecientes a los grados de primero y segundo de primaria, de los cuales fueron participes en el estudio 7 informantes claves.

Los criterios que se tuvieron en cuenta para la participación de la investigación son los siguientes (Tabla 1):

TABLA 1. Criterios de inclusión y exclusión.

\begin{tabular}{cc}
\hline Criterios de Inclusión & Criterios de exclusión \\
\hline $\begin{array}{c}\text { Docentes del colegio Manuel Antonio } \\
\text { Rueda Jara del Municipio de Villa del } \\
\text { Rosario }\end{array}$ & $\begin{array}{c}\text { Docentes de otras instituciones del } \\
\text { municipio de Villa del Rosario. }\end{array}$ \\
$\begin{array}{c}\text { Docentes de los grados primero y } \\
\text { segundo. }\end{array}$ & $\begin{array}{c}\text { Docentes de grados diferentes a } \\
\text { primero y segundo. }\end{array}$ \\
\hline
\end{tabular}

Fuente: Autores.

Se tuvo en cuenta para el desarrollo del estudio, cuatro procesos fundamentales; en el primer proceso se lleva a cabo la estrategia de meta plan grabado en audio y video, la cual tuvo como fin recopilar gran parte de información y datos proporcionados por las participantes del mismo, y sus opiniones acerca de las estrategias tenidas en cuenta para la cartilla de intervención fonoaudiológica indirecta.

Revista Científica Signos Fónicos, 2020, 6 (2): 1-23. ISNN 2422-1716. 
En el segundo proceso, se llevó a cabo una encuesta semiestructurada mixta, con el fin de recoger las opiniones de los docentes acerca de las estrategias planteadas, con una calificación de acuerdo a la metodología de la escala Likert (27), acompañada de una pregunta abierta con la cual se pretende identificar las sugerencias o recomendaciones de las docentes ante las estrategias de intervención indirecta desarrolladas.

El análisis de la información recopilada en el meta plan conforma el tercer proceso, teniendo en cuenta el software de análisis de datos cualitativos denominado Atlas ti versión 7.5 .18 (28), por medio del cual se realiza la codificación abierta, selectiva y axial de los datos obtenidos.

- Codificación abierta: proceso analítico por medio del cual se identifican conceptos y se descubren en los datos sus propiedades y dimensiones (29), (30).

- Codificación axial o Selectiva: se trata del proceso de relacionar las categorías a sub -categorías denominado "axial" porque la codificación ocurre alrededor del eje de una categoría, y enlaza las categorías en cuanto a sus propiedades y dimensiones (29), (31).

Finalmente el cuarto proceso se lleva a cabo con la corrección y modificación del primer diseño de las estrategias de intervención indirecta de acuerdo a las sugerencias dadas por las docentes, este proceso se realizó, por medio de la utilización del software denominado Adobe Indesign el cual es una aplicación para diseño editorial, se puede realizar desde proyectos sencillos, hasta creaciones más complejas tales como revistas, libros convencionales, electrónicos, periódicos entre otros, en este estudio se utilizó Adobe Indesign para maquetar el diseño de las estrategias para la lectoescritura en formato revista destinada para impresión (32).

\section{RESULTADOS}

A partir de las encuestas realizada se identifica la percepción de cada docente acerca de las estrategias de intervención fonoaudiológica indirecta correspondientes a la lectoescritura mediante preguntas mixtas, en primer lugar, se tuvo en cuenta la escala Likert para el componente cuantitativo de la investigación donde la pregunta tenía por fin percibir el nivel de concordancia a partir de 1 (menor nivel de satisfacción) hasta 5 (máximo nivel de satisfacción). Teniendo en cuenta esto se encontró (Tabla 2):

TABLA 2. Nivel de concordancia.

\begin{tabular}{cccc}
\hline $\begin{array}{c}\text { Nivel De } \\
\text { Concordancia }\end{array}$ & Frecuencia & Porcentaje \\
\hline 4 & 3 & $42.8 \%$ \\
TOTAL & 5 & 4 & $57.1 \%$ \\
\hline
\end{tabular}

Fuente: Autores. 
Aguilar, Ayala et al. Revista Científica Signos Fónicos, 2020, 6(2): 1-23

Así mismo, a partir de una pregunta abierta, se consiguió que cada una de las docentes diera sugerencias de acuerdo a las estrategias que le fueron socializadas de lectura y escritura para primer y segundo grado socializadas, de esta manera a partir de la respuesta de siete docentes se generaron cinco categorías, las cuales surgieron de la agrupación y delimitación de cada respuesta obtenida (Tabla 3).

TABLA 3. Categorías generadas.

\begin{tabular}{lcc}
\hline \multicolumn{1}{c}{ Categorías } & Frecuencia & Porcentaje \\
\hline $\begin{array}{l}\text { Vinculación padres al proceso del } \\
\text { aprendizaje }\end{array}$ & 2 & $28.5 \%$ \\
$\begin{array}{l}\text { Dramatizaciones de cuentos o fabulas } \\
\text { cortas por parte del niño }\end{array}$ & 1 & $14.2 \%$ \\
$\begin{array}{l}\text { Uso de estrategias fonológicas para } \\
\text { fortalecer las rutas implicadas en la } \\
\text { lectoescritura de los fonemas p-q y d-b. }\end{array}$ & 2 & $28.5 \%$ \\
Dinámicas o juegos grupales & 1 & $14.2 \%$ \\
$\begin{array}{l}\text { Creación de aula de apoyo para mejorar el } \\
\text { proceso de la lecto escritura }\end{array}$ & 1 & $14.2 \%$ \\
TOTAL & 7 & $99.6 \%$ \\
\hline
\end{tabular}

Fuente: Autores.

Seguidamente, se realiza la formulación y reestructuración de las estrategias de lectoescritura mostradas a partir de los resultados de acuerdo al meta- plan, donde se tuvo en cuenta si la estrategia tiene alguna desventaja, requiere de alguna modificación o en lugar a esto, que fortalece y que permite cada uno de los procesos de lectoescritura (Tabla 4). 
TABLA 4. Resultados de Metaplan.

\begin{tabular}{|c|c|}
\hline \multicolumn{2}{|r|}{ Resultados de meta plan } \\
\hline Fortaleza & $\begin{array}{l}\text { - } \\
\text { - } \\
\text { - } \\
\text { - } \\
\text { - } \\
\text { Lortalecimiento académico del estudiante } \\
\text { escolares } \\
\text { - } \quad \text { El aprendizaje del lenguaje oral y escrito }\end{array}$ \\
\hline Desventaja & $\begin{array}{l}\text { - Falta de acompañamiento de los padres o acudientes para un mejor } \\
\text { desempeño } \\
\text { - } \quad \text { Indisciplina de los estudiantes que interrumpe el aprendizaje } \\
\text { - Grupos numerosos y falta de tiempo }\end{array}$ \\
\hline Permite & $\begin{array}{l}\text { - } \text { Reforzar el proceso de lectoescritura } \\
\text { - } \text { Reconocer sonidos y aplicación de estos en la lectura y escritura de las } \\
\text { palabras } \\
\text { - } \quad \text { Desarrollar habilidades en la lectoescritura de los estudiantes } \\
\text { - } \\
\text { - } \\
\text { - } \text { Que el niño lectura adecuada } \\
\text { - Ayudar al estudiante a pensar. }\end{array}$ \\
\hline Requiere & $\begin{array}{ll}\text { - } & \text { Material para ponerlas en practica } \\
\text { - } & \text { Enseñanza más personalizada a cada docente } \\
\text { - } & \text { Mas creatividad } \\
\text { - } & \text { Apuntar a los estudiantes con más necesidades } \\
\text { - } & \text { Tiempo } \\
\text { - } & \text { Dedicación } \\
\text { - } & \text { Compromiso del padre y acompañamiento } \\
\text { - } & \text { Sean compartidas a los docentes con anticipación }\end{array}$ \\
\hline
\end{tabular}

Fuente: Autores.

Posteriormente, a partir de la grabación de audio obtenida de la socialización de las estrategias de lectoescritura a los docentes de primero y segundo grado, se obtuvieron los siguientes resultados a través del análisis cualitativo de los datos a partir del Software Atlas ti versión 7s (Tabla 5):

TABLA 5. Códigos in vivo y Constructo social. 


\begin{tabular}{|c|c|c|}
\hline \multicolumn{3}{|c|}{ Aguilar. Avala et al. Revista Científica Signos Fónicos. 2020. 6(2): 1-23 } \\
\hline $\mathrm{N}^{\circ}$ & CODIGO & TIPO \\
\hline 1. & Reconocimiento e interpretación de la lectura & Constructo Social \\
\hline 2. & Reglas de correspondencia y comunicación & Constructo Social \\
\hline 3. & $\begin{array}{c}\text { Reestructuración y transformación hacia nuevos } \\
\text { conocimientos }\end{array}$ & Constructo Social \\
\hline 4. & Extracción del significado del texto & Constructo Social \\
\hline 5. & Interacción con la lengua escrita & Constructo Social \\
\hline 6. & $\begin{array}{l}\text { Lengua escrita como medio para construir el } \\
\text { conocimiento }\end{array}$ & Constructo Social \\
\hline 7. & Teoría & Código in-vivo \\
\hline 8. & conciencia fonológica- Sonido & Constructo Social \\
\hline 9. & Consonante-vocal & Constructo Social \\
\hline 10. & Vocabulario & Código in-vivo \\
\hline 11. & Almacén visual & Constructo Social \\
\hline 12. & Silabeo & Código in-vivo \\
\hline 13. & Selección y organización de la información & Constructo Social \\
\hline 14. & Mecanización de la información & Constructo Social \\
\hline 15. & Procesos fonetico-fonologicos & Constructo Social \\
\hline 16. & Interiorizar el sonido & Constructo Social \\
\hline 17. & Problemas fonológicos & Constructo Social \\
\hline 18. & Esquema global de sonidos & Constructo Social \\
\hline 19. & Discriminación de palabras y letras & Constructo Social \\
\hline 20. & Diferenciación de sonidos & Constructo Social \\
\hline 21. & Reconocimiento de letras dentro de un texto & Constructo Social \\
\hline 22. & Lateralidad & Constructo Social \\
\hline 23. & Conversión de grafemas a sonidos & Constructo Social \\
\hline 24. & Combinación de grafemas & Constructo Social \\
\hline 25. & Eliminación de sonidos & Constructo Social \\
\hline 26. & Forma ortográfica de la palabra y significado & Constructo Social \\
\hline 27. & Reconocimiento inmediato de una palabra escrita & Constructo Social \\
\hline
\end{tabular}

Fuente: Autores.

Continuación TABLA 5. Códigos in vivo y Constructo social. 


\begin{tabular}{|c|c|c|}
\hline \multicolumn{3}{|c|}{ Aguilar. Avala et al. Revista Científica Signos Fónicos. 2020. 6(2): 1-23 } \\
\hline $\mathrm{N}^{\circ}$ & CODIGO & TIPO \\
\hline 28. & Pronunciación & Código in-vivo \\
\hline 29. & Ortografía & Código in-vivo \\
\hline 30. & Léxico & Constructo Social \\
\hline 31. & Reconocimiento de letras & Constructo Social \\
\hline 32. & Dinámicas o juegos grupales & Código in-vivo \\
\hline 33. & Estructuración de oraciones & Constructo Social \\
\hline 34. & Significado & Constructo Social \\
\hline 35. & Refuerzo & Código in-vivo \\
\hline 36. & Fonema & Constructo Social \\
\hline 37. & Grafema & Constructo Social \\
\hline 38. & Vinculación padres al proceso del aprendizaje & Código in-vivo \\
\hline 39. & Evolución con el tiempo & Código in-vivo \\
\hline 40. & $\begin{array}{c}\text { Creación de aula de apoyo para mejorar la dificultad en la } \\
\text { lectoescritura }\end{array}$ & Código in-vivo \\
\hline 41. & Intervención & Constructo Social \\
\hline
\end{tabular}

Fuente: Autores.

Codificación axial y selectiva: en la organización de los datos emergen cuatro categorías que representan la percepción docente sobre las estrategias de intervención indirecta a escolares de primer y segundo grado de primaria, encontrando (Tabla 6):

TABLA 6. Categoría emergente de lectura de primero de primaria.

\begin{tabular}{|c|c|c|c|c|}
\hline Fenómeno & Categoría & $\begin{array}{l}\text { Sub- } \\
\text { Categoría }\end{array}$ & Propiedades & Dimensiones \\
\hline \multirow{5}{*}{$\begin{array}{l}\text { Estrategias } \\
\text { de } \\
\text { intervención } \\
\text { indirecta }\end{array}$} & \multirow{5}{*}{$\begin{array}{l}\text { Primero de } \\
\text { primaria }\end{array}$} & \multirow{5}{*}{$\begin{array}{l}\text { Lectura } \\
\text { primero }\end{array}$} & $\begin{array}{l}\text { Reconocimiento } \\
\text { global de las palabras }\end{array}$ & $\begin{array}{l}\text { Reconocimiento e interpretación } \\
\text { de la lectura }\end{array}$ \\
\hline & & & $\begin{array}{l}\text { Conciencia } \\
\text { Metalingüística }\end{array}$ & $\begin{array}{l}\text { Reglas de correspondencia y } \\
\text { comunicación }\end{array}$ \\
\hline & & & $\begin{array}{l}\text { Incrementación de los } \\
\text { conocimientos previos }\end{array}$ & $\begin{array}{l}\text { Reestructuración } \\
\text { transformación hacia nuevos } \\
\text { conocimientos }\end{array}$ \\
\hline & & & Comprensión lectora & $\begin{array}{l}\text { Extracción del significado del } \\
\text { texto }\end{array}$ \\
\hline & & & $\begin{array}{l}\text { Interacción } \\
\text { significativa y } \\
\text { funcional del niño con } \\
\text { la lengua escrita como } \\
\text { medio para construir } \\
\text { los conocimientos }\end{array}$ & Interacción con la lengua escrita \\
\hline
\end{tabular}


Fuente: Autores.

Categoría 1: Las estrategias de escritura del grado primero se basan en una fundamentación teórica, así mismo, en estrategias de conciencia fonológica que incluyen las reglas fonología-sonido y consonantevocal.

Las estrategias de conciencia léxica según la percepción de los docentes deben abarcar más que el vocabulario el almacén visual del infante. por otra parte, la segmentación de silabas o silabeo deben incluirse como base de las estrategias de la conciencia silábica y las estrategias de memoria a corto plazo deben permitirle al estudiante la selección, organización y mecanización de la información.

La última estrategia a desarrollar corresponde al léxico fonológico en el cual los docentes surgieren la inclusión de procesos fonético-fonológicos e interiorización de sonido para prevenir posibles alteraciones a nivel fonológico (Tabla 7).

TABLA 7. Categoría emergente de escritura de primero de primaria.

\begin{tabular}{|c|c|c|c|c|}
\hline Fenómeno & Categoría & Sub-Categoría & Propiedades & Dimensiones \\
\hline \multirow{10}{*}{$\begin{array}{l}\text { Estrategias de } \\
\text { intervención } \\
\text { indirecta }\end{array}$} & \multirow{10}{*}{$\begin{array}{l}\text { Primero de } \\
\text { primaria }\end{array}$} & \multirow{10}{*}{$\begin{array}{l}\text { Escritura de } \\
\text { primero }\end{array}$} & $\begin{array}{l}\text { Fundamentación } \\
\text { teórica }\end{array}$ & Teoría \\
\hline & & & \multirow{2}{*}{$\begin{array}{l}\text { Conciencia } \\
\text { fonológica }\end{array}$} & Sonido \\
\hline & & & & Consonante-vocal \\
\hline & & & \multirow{2}{*}{ Conciencia léxica } & Vocabulario \\
\hline & & & & Almacén visual \\
\hline & & & Conciencia silábica & Silabeo \\
\hline & & & \multirow[t]{2}{*}{$\begin{array}{l}\text { Memoria a corto } \\
\text { plazo }\end{array}$} & $\begin{array}{l}\text { Selección y organización } \\
\text { de la información } \\
\text { Mecanización de la } \\
\text { información }\end{array}$ \\
\hline & & & & $\begin{array}{l}\text { Procesos fonetico- } \\
\text { fonologicos }\end{array}$ \\
\hline & & & \multirow[t]{2}{*}{ Léxico Fonológico } & Interiorizar el sonido \\
\hline & & & & Problemas fonológicos \\
\hline
\end{tabular}

Fuente: Autores. 
Aguilar, Ayala et al. Revista Científica Signos Fónicos, 2020, 6(2): 1-23

Categoría 2: La escritura en los escolares de primer grado, depende de la fundamentación teórica que apoye el proceso de aprendizaje, no obstante, existen otras propiedades como la conciencia fonológica, que encaminan al infante a un proceso de escritura eficiente, sin embargo, esta no es un proceso aparte, ya que se desarrolla según las reglas fonología-sonido y consonante vocal.

La conciencia léxica es una propiedad que no se reduce únicamente al vocabulario, sino que relaciona el almacén visual con la escritura.

Por otra parte, la conciencia silábica y memoria a corto plazo son propiedades que tienen su base en el silabeo y la selección, organización y mecanización de la información respectivamente.

Para terminar, el léxico fonológico en la escritura de los escolares abarca los procesos fonéticofonológicos lo que le permite al escolar interiorizar el sonido y de esta manera, intervenir en posibles problemas a nivel fonológico (Tabla 8).

TABLA 8. Categoría emergente de lectura de segundo de primaria.

\begin{tabular}{|c|c|c|c|c|}
\hline Fenómeno & Categoría & Sub-Categoría & Propiedades & Dimensiones \\
\hline \multirow{5}{*}{$\begin{array}{l}\text { Estrategias de } \\
\text { intervención } \\
\text { indirecta }\end{array}$} & \multirow{5}{*}{$\begin{array}{l}\text { Segundo de } \\
\text { primaria }\end{array}$} & \multirow{5}{*}{$\begin{array}{l}\text { Lectura de } \\
\text { segundo }\end{array}$} & \multirow[t]{2}{*}{ Método global } & $\begin{array}{l}\text { Esquema global de } \\
\text { sonidos } \\
\text { Discriminación de } \\
\text { palabras y letras }\end{array}$ \\
\hline & & & & $\begin{array}{l}\text { Diferenciación de sonidos } \\
\text { Reconocimiento de letras } \\
\text { dentro de un texto }\end{array}$ \\
\hline & & & $\begin{array}{l}\text { Reglas grafema- } \\
\text { fonema }\end{array}$ & $\begin{array}{l}\text { Lateralidad } \\
\text { Conversión de grafemas a } \\
\text { sonidos }\end{array}$ \\
\hline & & & & Combinación de grafemas \\
\hline & & & Ruta visual & $\begin{array}{l}\text { Eliminación de sonidos } \\
\text { Forma ortográfica de la } \\
\text { palabra y significado } \\
\text { Reconocimiento } \\
\text { inmediato de una palabra } \\
\text { escrita }\end{array}$ \\
\hline
\end{tabular}

Fuente: Autores.

Categoría 3: El proceso de lectura en los escolares de segundo grado se desarrolla a través de tres propiedades que corresponden al método global, reglas grafema-fonema y ruta visual.

Las sugerencias de los docentes indican que el método global debe abarcar el esquema global de sonidos, discriminación de palabras y letras, diferenciación de sonidos y reconocimiento de letras dentro de un texto.

La lateralidad, conversión de grafema a sonidos y combinación de dichos grafemas son sugerencias para incluir en las estrategias de regla grafema-fonema, la ruta visual es una estrategia que se desarrolla siguiendo los procesos de eliminación de sonidos, teniendo en cuenta la forma ortográfica de la palabra y su significado, lo que según el docente permitirá el reconocimiento inmediato de una palabra escrita por parte de un escolar (Tabla 9). 
TABLA 9. Categoría emergente de escritura de segundo de primaria.

\begin{tabular}{|c|c|c|c|c|}
\hline Fenómeno & Categoría & Sub-Categoría & Propiedades & Dimensiones \\
\hline \multirow{9}{*}{$\begin{array}{l}\text { Estrategias de } \\
\text { intervención } \\
\text { indirecta }\end{array}$} & \multirow{9}{*}{$\begin{array}{l}\text { Segundo de } \\
\text { primaria }\end{array}$} & \multirow{9}{*}{$\begin{array}{l}\text { Escritura de } \\
\text { segundo }\end{array}$} & \multirow{4}{*}{ Procesos léxicos } & Pronunciación \\
\hline & & & & Ortografía \\
\hline & & & & Léxico \\
\hline & & & & $\begin{array}{l}\text { Reconocimiento de } \\
\text { letras }\end{array}$ \\
\hline & & & Planificación y & $\begin{array}{l}\text { Dinámicas o juegos } \\
\text { grupales }\end{array}$ \\
\hline & & & Generación de ideas & $\begin{array}{l}\text { Estructuración de } \\
\text { oraciones }\end{array}$ \\
\hline & & & Organizar las ideas & Significado \\
\hline & & & Procesos motores & Fonema \\
\hline & & & Estructura sintáctica & Grafema \\
\hline
\end{tabular}

Fuente: Autores.

Categoría 4: Las estrategias de intervención indirecta para la escritura del grado segundo incluyen procesos léxicos que deben fundamentarse en actividades de pronunciación, ortografía, léxico y reconocimiento de letras a su vez, las estrategias de planificación y generación de ideas deben generarse a partir de dinámicas o juegos grupales que permitan reflejar estas ideas en una oración escrita.

Según lo anterior la siguiente estrategia para favorecer este proceso es la organización de las ideas de acuerdo al significado de las palabras, teniendo en cuenta la correspondencia fonema-grafema para construir finalmente una estructura sintáctica.

Finalmente, de acuerdo a las diferentes codificaciones realizadas por medio del software Atlas ti 7 , surgieron las redes semánticas, las cuales en su conjunto constituyen la percepción que tienen las docentes de acuerdo a las estrategias de intervención indirecta para la lectoescritura en los grados de primero y segundo (Figuras 1,2,3,4). 


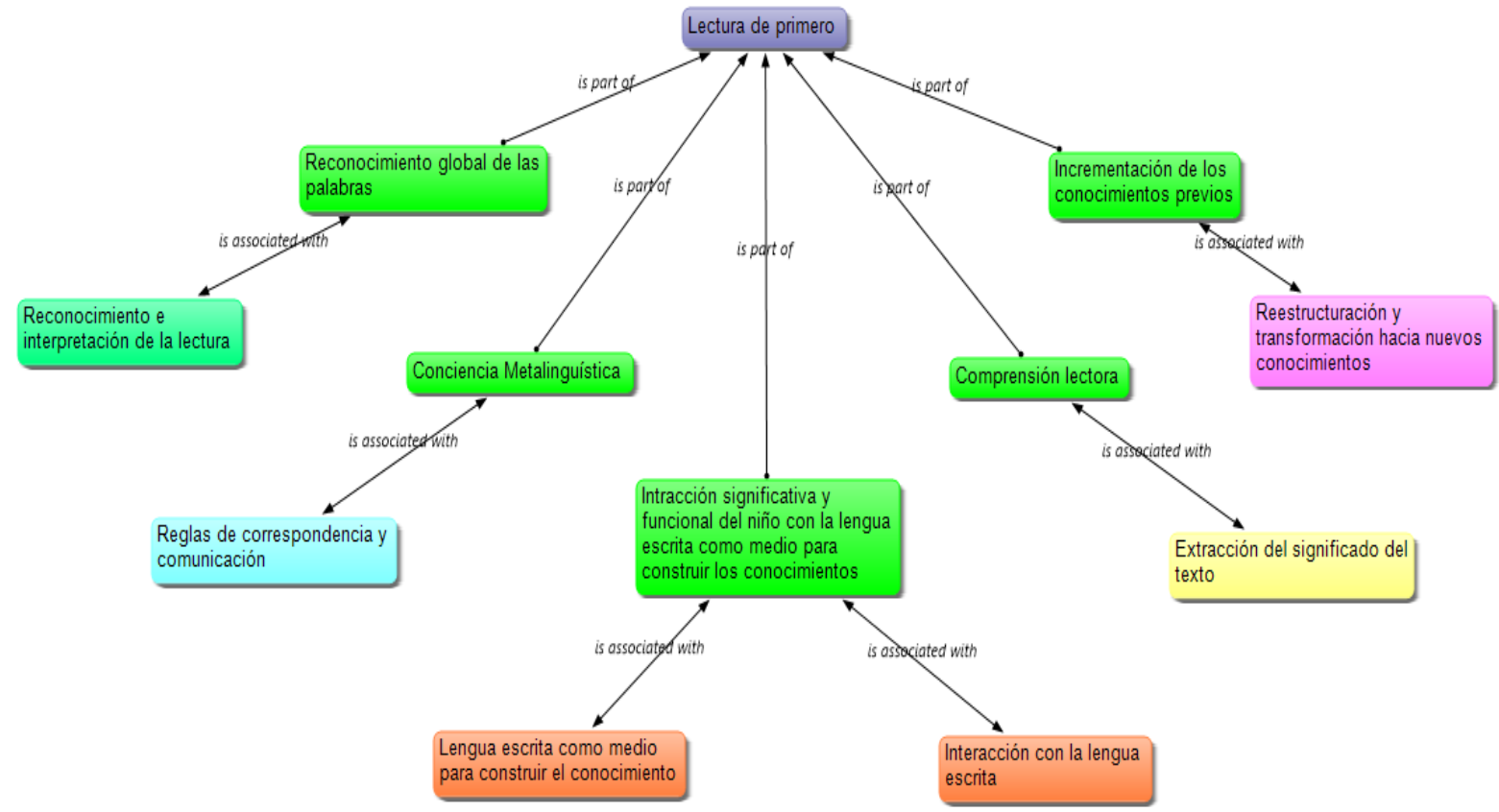

Figura 1. Red semántica de la categoría Lectura de Primero.

Fuente: Autores.

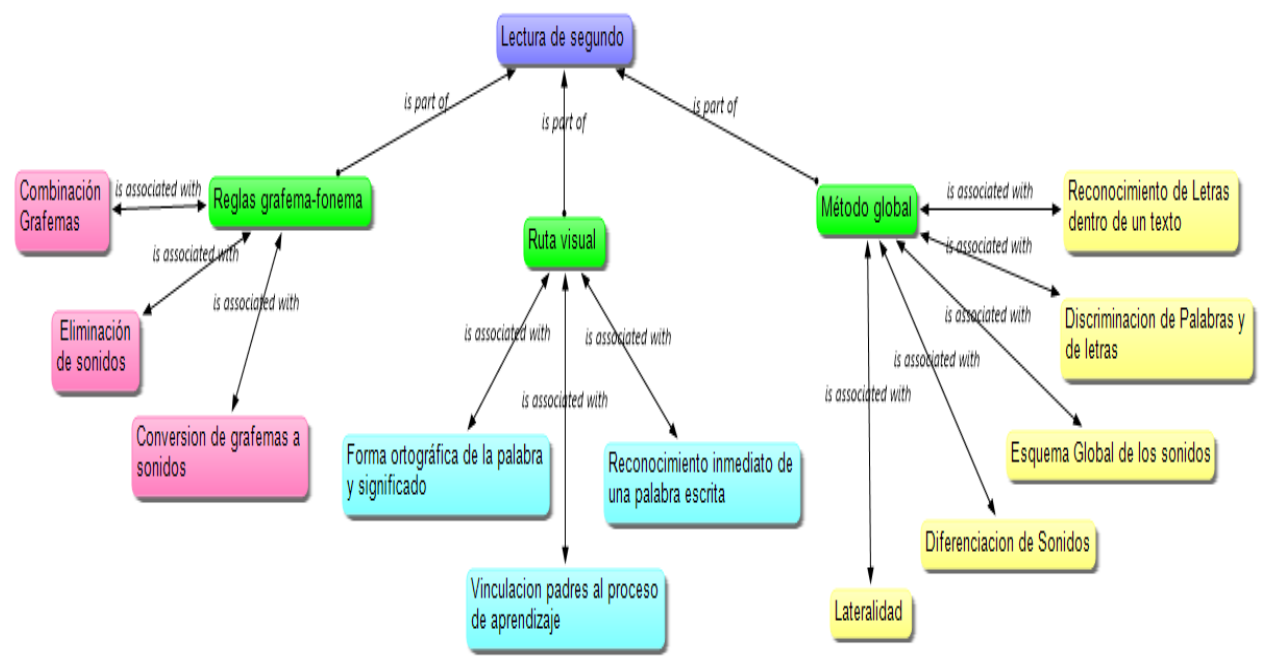

Figura 2. Red semántica de la categoría Lectura de Segundo.

Fuente: Autores. 


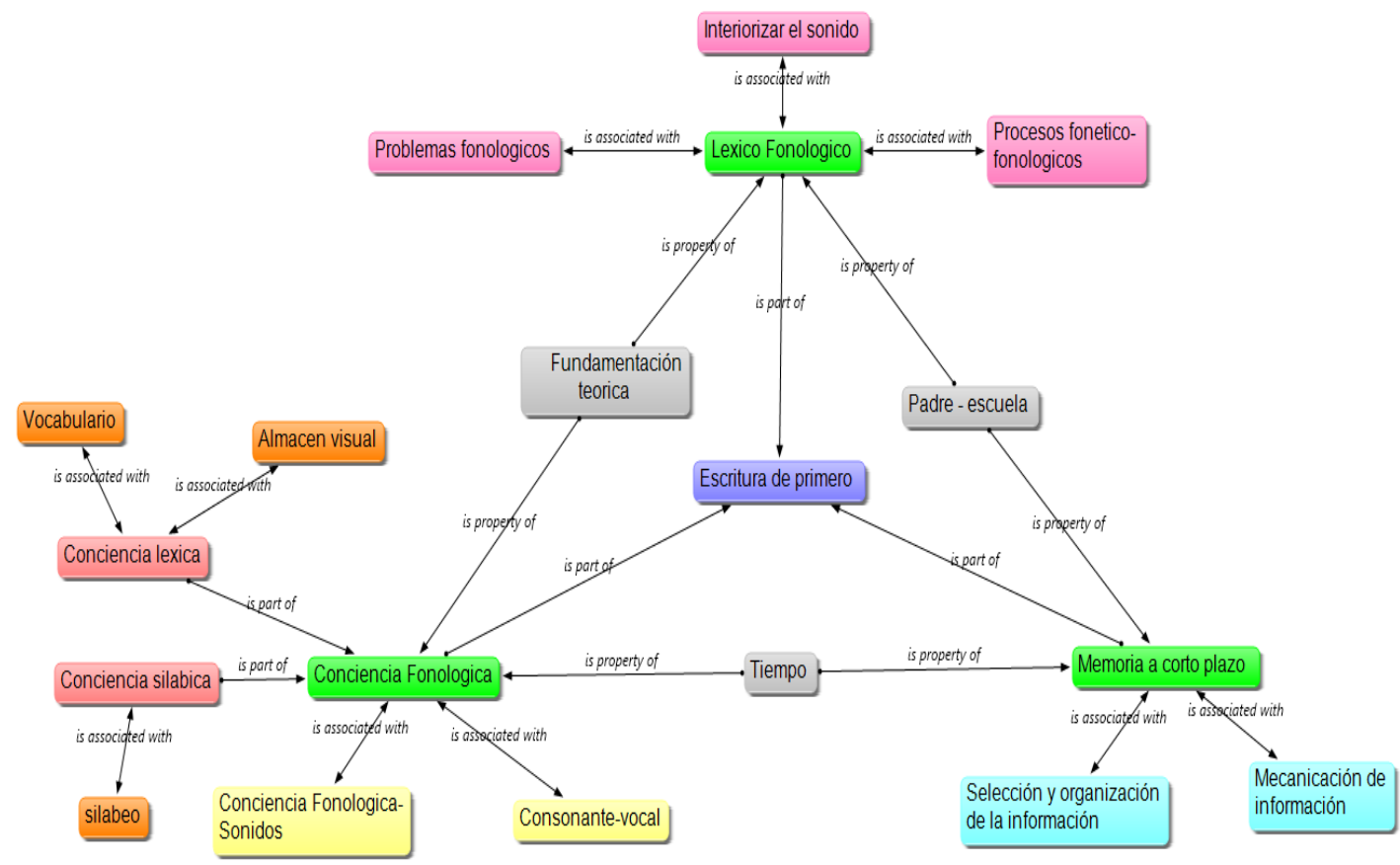

Figura 3. Red semántica de la categoría Escritura de Primero.

Fuente: Autores.

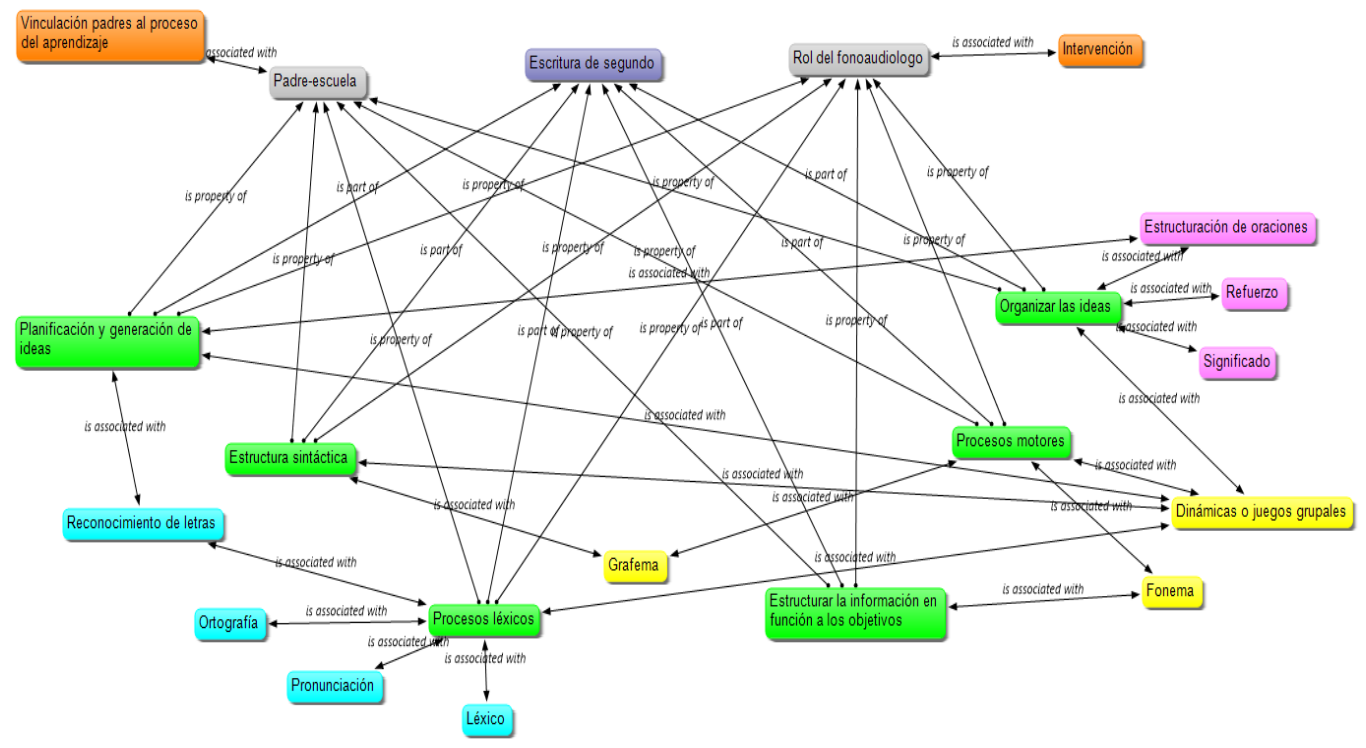

Figura 4. Red semántica de la categoría Escritura de Segundo.

Fuente: Autores. 


\section{Aguilar, Ayala et al. Revista Científica Signos Fónicos, 2020, 6(2): 1-23}

\section{ANÁLISIS Y DISCUSIÓN}

Se entiende por percepción, el proceso cognitivo de la conciencia que tiene en cuenta el reconocimiento, interpretación y significado de un tema propuesto, concluyendo con un juicio u opinión (33), por otra parte, la percepción de un individuo sobre un fenómeno, depende del conocimiento que este mismo tenga sobre el tema, lo que le permitirá dar una impresión o inferencia (34). Según esto, las encuestas realizadas permitieron recopilar la percepción de los docentes en relación a las estrategias de intervención indirecta, se obtuvo un nivel de concordancia de un $99 \%$ lo que indica un alto nivel de satisfacción con las estrategias planteadas. Además, las respuestas a la pregunta abierta se organizaron en 5 categorías que agrupan las sugerencias para las estrategias en las cuales predomina la importancia de la vinculación de los padres de familia al proceso de aprendizaje y el uso de estrategias fonológicas para fortalecer las rutas implicadas en la lectoescritura, no obstante los cuentos o fabulas, dinámicas o juegos grupales y la creación de un aula de apoyo son de menos relevancia, sin embargo, son vitales para el desarrollo efectivo de la lectoescritura.

La lectoescritura es un proceso y una estrategia (35) que se constituye en un aspecto muy importante en las decisiones que deben tomar las personas que tienen a cargo la gestión de una organización, en la que hay recursos de todo tipo que deben ser utilizados en forma óptima para cumplir con las políticas y metas trazadas; para el aprendizaje y a su vez, un sistema de comunicación y meta cognición que se aplica desde los inicios de la escolaridad de acuerdo a las habilidades de cada escolar con el objetivo de lograr un adecuado procesamiento fonológico y expresión social (36). Según lo anterior, la implementación de distintas estrategias para el desarrollo de la lectoescritura basadas en el juego permiten atender las dificultades del niño en la expresión oral o escrita, generar un aprendizaje significativo ya que capta la atención de los alumnos (37), fomentar el desenvolvimiento social y comunicativo con sus pares, acrecentar procesos de percepción, memoria, cognición, entre otros y de esta manera satisfacer el desarrollo educativo integral del estudiantado (38). Para complementar lo planteado, los docentes sugieren la creación de un aula de apoyo como una ruta alternativa para atender de manera integral las necesidades de cada escolar y que cuente con el ejercicio de un equipo disciplinar que involucre el fonoaudiólogo como guía principal para el proceso comunicativo.

Por otra parte, los resultados obtenidos a partir del meta plan en relación a la percepción de los docentes con respecto a las estrategias planteadas (39), indican que fortalecen el desarrollo del lenguaje oral y escrito y los procesos de adquisición fonemática (40), lo que a su vez influye positivamente en el crecimiento académico del estudiante, además, cabe resaltar, que los docentes indicaron que las estrategias permiten reforzar el proceso de lectoescritura que es una habilidad que toda persona debe desarrollar para integrarse a una sociedad (41).

Al proponer el reconocimiento y aplicación de sonidos para la ejecución de este proceso, asimismo, permiten que el niño tenga un proceso de asimilación e integración de los sonidos del habla eficiente, lo que le ayudara a desarrollar su pensamiento y distintas habilidades que concluirán en un proceso de lectura lo cual es una actividad que consiste en interpretar y descifrar, mediante la vista, el valor fónico de una serie de signos escritos(42) y la escritura es una codificación sistemática mediante signos gráficos que permite registrar con gran precisión el lenguaje adecuado (43). Sin embargo, otro contrapunto a tener en cuenta reside en la falta de acompañamiento de los padres para un mejor desempeño del escolar, en la cantidad de alumnos que conforman un aula de clases, su comportamiento y el tiempo asignado para llevar a cabo una estrategia específica (44).

El juicio de los docentes denota que además existen varios requerimientos a tener en cuenta como lo son los materiales para poner en práctica las estrategias planteadas, la dedicación tanto de los mismos Revista Científica Signos Fónicos, 2020, 6 (2): 1-23. ISNN 2422-1716. 
Aguilar, Ayala et al. Revista Científica Signos Fónicos, 2020, 6(2): 1-23

docentes como el acompañamiento de la familia y el apoyo extracurricular personalizado e individual por parte de un equipo interdisciplinar que evalué y atienda las necesidades específicas de los estudiantes que presenten mayor dificultad para integrar estos procesos (45). El fonoaudiólogo como actor principal para el desarrollo de la lectura y la escritura, cumple el rol de asesor y colaborador con el equipo docente en el contexto escolar (46).

El contexto escolar pretende dar soluciones a necesidades educativas especiales a aquellos alumnos que lo necesiten (47) y esta realidad ayuda a que el fonoaudiólogo se involucre en este ambiente donde atiende necesidades asociadas a la comunicación, es decir el rol fonoaudiológico en escuela demanda un cambio en la colaboración a un maestro tutor, apoyo curricular e intervención a los alumnos (48). La atención a niños o jóvenes desde el campo de la fonoaudiología dentro de una institución precisa en la no atención individual o específica a los niños sino que por el contrario esta sea de manera colectiva y además en la orientación a padres de familia y docentes siendo estos un pilar fundamental en la construcción de conocimiento en el ámbito educativo (49).

El trabajo fonoaudiológico representa un acercamiento al aula aprovechando la relación existente entre el lenguaje y el aprendizaje favoreciendo el desarrollo integral del alumno (39), paralelo a esto la colaboración o ejercicio con los maestros apoyándose en el curriculum y las situaciones naturales que tienen lugar en la escuela. El maestro del aula es considerado el recurso principal para la instrucción de los alumnos en procesos como lo son la lectura y escritura a través de la aplicación de estrategias que promuevan el desarrollo de estos procesos de acuerdo al grado del escolar (50).

Las estrategias de lectura en los escolares de primer y segundo grado de primaria deben basarse en el reconocimiento global de las palabras, es decir, en el método global, siendo este el mejor ya que contempla las características de pensamiento del niño que ingresa en primer grado porque a esta edad ( 5 a 6 años) percibe sincréticamente cuanto le rodea y no siente espontáneamente la necesidad de analizar las partes de un todo. Por otra parte, el desarrollo de las habilidades metalingüísticas (51) implica a su vez la conciencia fonológica como base del aprendizaje de la lectura inicial según lo demuestran varios estudios (52).

En el caso de los escolares de segundo grado, la diferencia en las estrategias a utilizar radica en que son más conscientes con respecto al esquema de sonidos que conforman el lenguaje oral, esto se define como conciencia de las unidades fonológicas del lenguaje hablado y se relaciona con la habilidad de reflexionar conscientemente sobre los segmentos fonológicos del lenguaje oral y la capacidad del infante para manipular dichas unidades (53).Así pues, la percepción de los docentes sobre las estrategias de lectura para los escolares de segundo grado indica que debe existir una ejecución competente de las reglas de conversión grafema fonema y la integración de la ruta visual, esta última, es clave para crear conciencia segmental y aprender la correspondencia letra-sonido (54).

La escritura en primer grado, se fundamenta en el ejercicio de distintas propiedades como lo son la conciencia fonológica, léxica, silábica y la memoria a corto plazo. La conciencia fonológica es la representación del sistema fónico, cabe resalar que varias investigaciones sugieren (55).

que la representación que el escolar tiene de los fonemas no se encuentra suficientemente adquirida en este nivel educativo y que en cambio, la conciencia silábica si lo está (56).En contraste, las estrategias de escritura para los escolares de segundo grado, implican el desarrollo de procesos léxicos, planificación, organización de ideas, estructuración de la información y procesos motores (57) ya que en este grado, el infante presenta una completa representación de los fonemas de la lengua y logra planificar un mensaje para transmitirlo a través de una estructura sintáctica en la cual incluye elementos 
Aguilar, Ayala et al. Revista Científica Signos Fónicos, 2020, 6(2): 1-23

ortográficos, que son el uso correcto de las letras y los signos para una correcta expresión escrita(58) y a su vez, adquiere habilidades y destrezas sobre los patrones motores que simbolizan cada grafía(59); esta no es más que la representación concreta de los sonidos de una lengua (60).

En este panorama, las estrategias de intervención indirecta según la percepción de los docentes están acorde a las necesidades específicas de la educación básica en los grados primero y segundo ya que permiten al fonoaudiólogo a través del docente desarrollar las habilidades comunicativas básicas para leer y escribir (4). A partir de esto, el docente tiene el objetivo de estimular al niño a través de estrategias otorgadas por el profesional, que le permitirán favorecer el descubrimiento de las funciones sociales de la escritura y de la lectura, es decir, que el alumno reconozca el uso de estos procesos en situaciones que reflejen el placer de disfrutar de una lectura entretenida, saber encontrar información útil para jugar (61), ya que este ha sido considerado como una actividad de carácter universal, común a todas las razas, en todas las épocas y para todas las condiciones de vida. En ese sentido, los gustos y las costumbres en todo, aprender o trabajar, organizar ideas y no recargar la memoria, entre otras (52).

\section{CONCLUSIONES}

La percepción de los docentes con respecto al diseño de estrategias de intervención fonoaudiológica indirecta tiene un nivel máximo de satisfacción ya que cumple con los criterios establecidos según los derechos básicos de aprendizaje y las distintas rutas con base teórica que favorecen estos procesos en edades tempranas.

Las estrategias fueron consideradas una herramienta guía que servirá de apoyo para mejorar el proceso de enseñanza y aprendizaje del estudiante en la lectoescritura. Dichas estrategias serán usadas intencional y flexiblemente por el docente, durante el desarrollo de los contenidos del aula para favorecer el lenguaje oral y escrito y reforzar el aprendizaje de nueva información.

A partir de los resultados obtenidos se crearon 5 categorías que permiten identificar la apreciación de los docentes de acuerdo a la intervención indirecta realizada por fonoaudiólogos dentro del rol escolar, esta población considera que el apoyo que se debe brindar en este campo debe de forma directa e involucrando a la familia. 


\section{Aguilar, Ayala et al. Rev
REFERENCIAS BIBLIOGRÁFICAS}

1. Álvarez B. Fonoaudiólogos y profesores: lo que hay detrás de los problemas al trabajar juntos. Ciencias la Salud. 2006;4(1):79-82.

2. García J. Comunicación, Calidad de Vida y Evaluación en Programas de Salud. Razón y palabra, 2004; 40, :4. Disponible en: https://dialnet.unirioja.es/servlet/articulo?codigo=962392

3.Osses Bustingorry S, Mora SJ. Metacognicion: un camino para aprender a aprender. Estud Pedagógicos. 2008; 34.

4. Álvarez L, Osorno M. Rol del fonoaudiólogo en Instituciones educativas en ciclos preescolar y básica primaria. Areté. 2012;12(1):33-42.

5. Montealegre R, Forero LA. Desarrollo de la lectoescritura: adquisición y dominio. Acta Colombiana de Psicología. 2015; 9: 25-40.

6.. Kirk S.A, McCarthy J.J. Kirk WDK, Adaptadores: S. Ballesteros y A. Cordero (Dpto. de I+D+i de TEA Ediciones). ITPA. Test Illinois de Aptitudes Psicolingüísticas. 1 Ed. Tea Ediciones S.A, 2011.

7. Núñez MP, Santamarina M. Prerrequisitos para el proceso de aprendizaje de la lectura y la escritura conciencia fonológica y destrezas orales de la lengua. Lengua y Habla. 2014:18, 72-92.

8. Rodríguez VMA, Méndez IA, Díaz NC. The connection between oral language and early reading: a paradigm change. Revista de Logopedia, Foniatria y Audiologia. 2018;38(3):93-4.

9. López-Contreras, Luísa-Fernanda. Grado De Severidad De Disfonía A Través Del Análisis Acústico Vocal En Docentes De Dos Instituciones De La Estrategia De Atención A La Primera Infancia. Revistas Científica Signos Fónicos. 2019: 5 (1). 1-9, https://doi.org/10.24054/01204211.v1.n1.2019.3976.

10. Cáceres-Mansilla, Luz-Amparo. Sindrome de Burnout, Trastornos del Sueño y su asociación con las alteraciones vocales en docentes de primera infancia. Revistas Científica Signos Fónicos. 2019: 5 (1). 10-19, https://doi.org/10.24054/01204211.v1.n1.2019.3977

11. Rodríguez E. Rol mediador del docente en el proceso de aprendizaje. [Tesis]. Universidad de Carabobo (Venezuela), 2016.

12. Cárdenas K, Guevara Y. Comprensión lectora en alumnos de secundaria. intervención por niveles funcionales. J Behav Heal Soc Issues. 2013; 5:67-83.

13. Salsa AM, Peralta OA. La "lectura" de material ilustrado. Revista Intercontinental de Psicología y Educación. 2001; 3 (1), 49-56.

14. Montealegre R, Forero L A. Desarrollo de la lectoescritura: adquisición y dominio. Act.Colom. Psicol. 2006; 9(1): 25-40.

15. Khan K, Mauro M, Migliorati P, Leonardi R. Gender and Expression Analysis Based on Semantic Face Segmentation. Image Analysis and Processing - ICIAP.2017;10485 (1): 37-47. DOI: 10.1007/978-3-319-68548-9_4

16. Francés FJ, Alaminos A, Penalva C, Santacreu OA. La investigación participativa: métodos y técnicas.1 Ed. Ecuador: PYDLOS Ediciones, 2015.

17. Cuetos F. Lectura y escritura de palabras a través de la ruta fonológica. Infanc y Aprendiz J Study Educ Dev. 1989; 45:71-84. 
Aguilar, Ayala et al. Revista Científica Signos Fónicos, 2020, 6(2): 1-23

18. María EV, López E, Zabala MT. Implicancias de diferentes modelos de la ciencia en la comprensión lectora. Fundamentos en Humanidades.2005; VI, (12):79-93.

19. Navarro-Criollo; Paola-Lorena. Validación de indicadores de perturbación de Jitter y Shimmer en docentes de primera infancia. Revistas Científica Signos Fónicos. 2019: 5 (1). 20-29, https://doi.org/10.24054/01204211.v1.n1.2019.3978

20. Manuel J, Calderón S, Javier F, Acosta C, Javier V, Mercado S, et al. Derechos Básicos de Aprendizaje. (actualizado 2016; el acceso en el año 2019 Julio 20).

21.Cortes D. DBA (Derechos Básicos de Aprendizaje) | Colombia Aprende. (actualizado 2015; el acceso en el año 2019 Julio 3).

22. Pacheco A, Blanco, Mercedes. Metodología mixta: su aplicación en México en el campo de la demografía. Estudios Demográficos y Urbanos.2015; 30(3): 725- 770.

23. Uribe M, Covarrubias K Y, Uribe Jl. La metodología mixta en un estudio sobre los ex braceros colimenses: una experiencia interdisciplinaria para comprender una realidad compleja. Estudios sobre las Culturas Contemporáneas. 2009; 15 (30), 123-154.

24. Rodríguez M, Mendivelso F. DISEÑO DE INVESTIGACIÓN DE CORTE TRANSVERSAL. Revista Médica Sanitas Rev.2018; 21 (3): 141-146.

25. Ander-Egg E. Repensando la Investigación-Acción Participativa. (actualizado 2003; el acceso en el año 2019 Julio 18).

26. Colmenares M. Investigación-acción participativa: una metodología integradora del conocimiento y la acción. Rev Latinoam Educ.2012;3(1):102-15.

27. Carvajal-Villamizar,Yuliana-Mercedes. Actitudes de los docentes y estudiantes hacia las personas con discapacidad en una institución pública. Revistas Científica Signos Fónicos. 2019: 5 (1). 30-44, https://doi.org/10.24054/01204211.v1.n1.2019.3979

28. Abizanda P, Romero L. Innovación en valoración funcional. Rev Esp Geriatr Gerontol. 2006; 41:27-35.

29. Nevárez A, Medina JA, Nevárez MM, Vargas J, Consdtance D, Guzmán DA, et al. Attention to Rhotacism Language Problem by Oral Surgery and Vibrostimulatory Therapy: A Case Report. Int J Odontostomatol. 2013;7(1):25-8.

30. Martínez-Siza, Diana-Carolina. Creencias de los docentes acerca de la educación inclusiva: un estudio fenomenológico. Revistas Científica Signos Fónicos. 2019: 5 (1). 45-59, https://doi.org/10.24054/01204211.v1.n1.2019.3980

31. VallésA, Vila J, García Ó, Casanovas M. Fiabilidad y validez del cuestionario observacional de las conductas de alimentación en neonatos prematuros (COCANP). Rev Logop Foniatría y Audiol. 2018;38(4):155-61.

32. Romero E. Indesign ¿Qué es y para qué sirve? Revista Digital. (actualizado 2015; el acceso en el año 2019 Julio 1).

33. Beleño-Melo; Betsy-Patricia. Calidad de vida en la inclusión educativa de niños y adolescentes con discapacidad intelectual. Revistas Científica Signos Fónicos. 2019: 5 (1). 60-72, https://doi.org/10.24054/01204211.v1.n1.2019.3982 
34. Castilla C. Enfoques teóricos sobre la percepción que tienen las personas. Horizontes Pedagógicos. 2006;8(1):9-22.

35.Contreras E F. El concepto de estrategia como fundamento de la planeación estratégica. Pensamiento \& Gestión. 2013; 35(2). 152-181

36. Ronquillo S. Iniciación a la lectoescritura a través del juego didáctico. [Tesis de pregrado]. Universidad Rafael Landívar (Quetzaltenango);2017.

37. Montero B. Experiencias Docentes Aplicación de juegos didácticos como metodología de enseñanza: Una Revisión de la Literatura Application of educational games as a teaching: A Literature Review. 2017; 7:92.

38. Moreno J B, Rodríguez M. L. Juegos didácticos como recurso para fortalecer la lecto-escritura en niños y niñas de tercer grado de primaria. [Tesis de pregrado]. Fundación Universitaria Los Libertadores (BOGOTÁ); 2017.

39. Achury S, Cifuentes I M, Lasso C, Páez S. Fonoaudiología en la estrategia de atención integral a la primera infancia de cero a siempre. Universidad del valle (Santiago de Cali); 2015.

40. Bigot M. Apuntes de lingüística antropológica. (actualizado 2010; el acceso en el año 2019 Julio 9).

41. Fe y Alegría. Aprendizaje de la lectoescritura. (actualizado 2010; el acceso en el año 2019 Julio 25).

42. Zuleta E. Sobre la lectura. (actualizado 1982; el acceso en el año 2019 Julio 25).

43.Perea M. Desarrollo didáctico de la escrita. CAUCE. Revista Internacional de Filología, Comunicación y sus Didácticas 2014; 36-37.

44. Lan Y T, Blandón DM, Rodríguez M M, Vásquez L E. Acompañamiento familiar en los procesos de aprendizaje. [Tesis de pregrado]. Universidad de San Buenaventura (Medellín); 2013.

45. Vera L, Mazadiego T. Una perspectiva sobre las actitudes y el deber ser de los docentes en el aula escolar. Revista de Educación y Desarrollo.2010; 14(2): 53-58.

46. Gasteiz V. Desarrollo del lenguaje y de la comunicación en el marco de un modelo inclusivo de intervención. IV. Serie. País Vasco; 2006.

47. SEP. Licenciatura en Educación Física Plan de Estudios. 1 Ed. Ciudad de México: Secretaría de Educación Pública;2018.

48.Castejón LA, España Y. La colaboración logopeda-maestro: hacia un modelo inclusivo de intervención en las dificultades del lenguaje. Rev Logop Foniatría y Audiol. 2004; 24(2):55-66. doi.org/10.1016/S0214-4603(04)75781-2

49. De León Sánchez B. La relación familia-escuela y su repercusión en la autonomía y responsabilidad de los niños/as. XXII Congreso Internacional de la Teoría de la Educación,2011.

50. Moran M, MSc. Vera LY, Morán MR. Los trastornos del lenguaje y las necesidades educativas especiales. consideraciones para la atención en la escuela. Revista Universidad y Sociedad. Universidad de Cienfuegos; 2009; 9 (3): 191-197.

51. Matalinares ML, Diaz G. Habilidades meta lingüísticas y comprensión en niños de primer grado de las ciudades de lima y Huancayo. Revista de Psicologia.2007. 
52. Flores CA, Martín M. El aprendizaje de la lectura y escritura en educación Inicial. Sapiens Rev Univ Investig. 2006;7(1):69-79.

53. Bizama M M, Arancibia G B, Sáez K. Intervención Psicopedagógica Temprana en Conciencia Fonológica como Proceso Metalinguistico a la base de la Lectura en niños de 5 a 6 años socialmente vulnerables. Estud pedagógicos. 2013;39(2):25-39.

54. Elisa Pa, López B. Tema 2. Sistema fonológico español. (el acceso en el año 2019 Julio 25).

55. Muñoz Y, Melenge J. La conciencia fonológica en el aprendizaje de la lectura convencional en un grupo de niños de 5 a 8 años. Revista de Investigaciones. 2017;17(29), 16-31. DOI: http://dx.doi.org/10.22383/ri.v17i29.85

56. Fraca De Barrera L. La conciencia fonológica silábica y el aprendizaje de la lengua escrita. Lectura y vida. 1998.

57. Vidigal C. Desarrollo motor y aprendizaje de la escritura en niños de 5 años. [Tesis de Posgrado]. Universidad de la Rioja ( España);2014.

58. Lahuerta J, De D, Belmonte IA. practica ortografía. Sociedad General Española de Librería, S. A. (acceso en el año 2019 Julio 24).

59. Tobeña C. Orientadora del IES Lucas Mallada procesos cognitivos de la escritura. cuando la tarea de escribir se lleva a cabo con dificultad: trastorno del aprendizaje de la escritura. procesos cognitivos y escritura. (acceso en el año 2019 Julio 24).

60. Salvador A. La adecuación entre grafía y fonema en los ortógrafos del siglo de oro. (acceso en el año 2019 Julio 24).

61. Minerva C. El juego: una estrategia importante. Educere.2002. 6(19), 289-296. 